\title{
Fano resonances in transport across a quantum well in a tilted magnetic field
}

\author{
Jens U. Nöckel* and A. Douglas Stone \\ Applied Physics, Yale University \\ P.O. Box 208284, Yale Station, New Haven CT 06520-8284 \\ * Current address: \\ Department of Physics, University of Oregon, 1371 E 13th Avenue, Eugene, OR 97403 \\ Received August 26, 1994; published in Physical Review B 51, 172219 (1995) \\ PACS: 03.80,11.20,72.20.D
}

\begin{abstract}
Asymmetric Fano resonances are predicted to occur in the linear electronic conductance across a shallow quantum well in a high magnetic field. The Fano lineshape results from the interference between direct transmission and transmission via a quasi-bound state in the well which is weakly mixed with the continuum by tilting the field. The resonance width increases with tilt angle but general S-matrix theory predicts that the lineshapes remain approximately unchanged upon rescaling of the field. This reflects a general property of Fano resonances which may be tested for the first time experimentally.
\end{abstract}

Resonances in electronic transport are of great interest in microstructures both as a basis for new device concepts and for characterization of heterostructures. Resonant tunneling devices operating at room temperature have become possible since three-dimensional heterostructures can be grown with atomic precision [1]. However recently a number of theoretical studies [2, 3, 4, 5, 6, 7, 8, 9, 10] have addressed resonances in quantum wire structures without large tunnel barriers. Typically these systems are calculated to exhibit asymmetric Fano resonances as opposed to the familiar symmetric Breit-Wigner (BW) lineshape. However no experiment has unambiguously observed asymmetric resonances in quantum wire devices (although there is some evidence for asymmetric resonances mediated by impurities [11] in the measurements of McEuen et al. [12]). It is likely that the quantum wire devices fail to demonstrate clear Fano resonances because of the lack of imperfections arising from the lack of a true epitaxial fabrication procedure for these devices[13]. Since the Fano lineshape represents the most general form for an isolated resonance it would be interesting to find such resonances experimentally in electronic transport and test aspects of scattering theory inaccessible in standard scattering experiments on atoms and nuclei. To this end we will show below that a certain type of 3D heterostructure in a tilted magnetic field should exhibit Fano resonances just as in the ideal quantum wire systems. However given the precision of heteroepitaxy it is much more likely that the experiments on such heterostructures will yield results consistent with the theory for the ideal structure. 
Before describing our model for the proposed experiment in more detail we review the relevant results from S-matrix theory. A single pole of the S-matrix with small imaginary part generates an isolated resonance; if the background phase shift $\bar{\theta}$ in the resonant scattering channel is roughly constant as the energy is varied through resonance then the most general resonant lineshape is described by the Fano function

$$
f_{q}(\epsilon)=\frac{(\epsilon+q)^{2}}{\epsilon^{2}+1}
$$

Here, $\epsilon=2\left(E-E_{R}\right) / \Gamma$ is the (dimensionless) energy from resonance and $\Gamma$ is the resonance width. In three-dimensional scattering from atoms or nuclei this lineshape is observed in scattering cross sections. Microstructures are expected to display the Fano lineshape in the conductance (transmission) [17]; in particular for a quantum wire with one propagating subband and an inversion- or reflection-symmetric scatterer one finds $T=\sin ^{2} \bar{\theta} f_{q}(\epsilon)$.

Fano resonances were originally studied in the case of inelastic (autoionizing) resonances of noble gases; although not obvious in Fano's original treatment, later work using a general S-matrix approach showed that the asymmetry parameter $q=-\cot \bar{\theta}$ in Eq. (1) is independent of the the coupling strength [14] $\Gamma$ so that each resonance will have a fixed $q$ even if $\Gamma$ is varied. Of course typically $\Gamma$ is not an experimentally controllable parameter in atomic scattering; however in the experiment described below $\Gamma$ should be tunable by varying the tilt angle and this prediction may be tested.

The physical origin of the asymmetric resonance lineshapes is the interference between direct scattering (described by the phase shift $\bar{\theta}$ ), and resonant scattering from a quasi-bound level of lifetime $\Gamma$ degenerate with the continuum. If the direct transmission is small then this interference is negligible and one recovers the symmetric Breit-Wigner lineshape (this corresponds to the limit $q \rightarrow \infty$ in Eq. (2)). This explains why the BW lineshape is always observed in purely $1 \mathrm{~d}$ resonant tunneling because the only means for making the lifetime long is to have opaque barriers which suppress the non-resonant transmission. To have a strongly asymmetric Fano lineshape it is necessary to have at least two scattering channels so that resonant and nonresonant transmission occur in parallel as two distinct processes. Then an electron entering the region where the quasi-bound state is localized does not necessarily enter that state itself. The background transmission can still be large even if the coupling to the quasi-bound level (which determines $\Gamma$ ) is small, (e.g. due to approximate symmetry).

We now illustrate these considerations for the case of our model. The heterostructure consists of an undoped quantum well of finite depth sandwiched between degenerately doped bulk emitter and collector regions with a magnetic field $\mathbf{B}$ tilted away from the normal to the layers (see Fig. 1). Far away from the well, transport in the direction of $\mathbf{B}$ reduces to a purely one-dimensional problem because the energy of the transverse motion is quantized into Landau levels (LL), leaving only the magnetic field direction for free motion. At the well interfaces, however, inter-LL scattering can take place. We will see below that in the mapping to the quantum wire problem the LL index $n=1,2, \ldots$ plays the role of the subband index and labels the different scattering channels that are needed to produce the Fano lineshape. When the magnetic field is exactly normal to the interfaces the Landau index will remain a good quantum number even across the interfaces and the problem is effectively $1 \mathrm{~d}$ with a transmission probability $T$ determined by the well potential. The well gives rise to a finite sequence of bound states which repeats below each LL threshhold $E_{n}$. The well width and depth may be chosen such that some bound states with LL index $n>1$ lie degenerate with the 


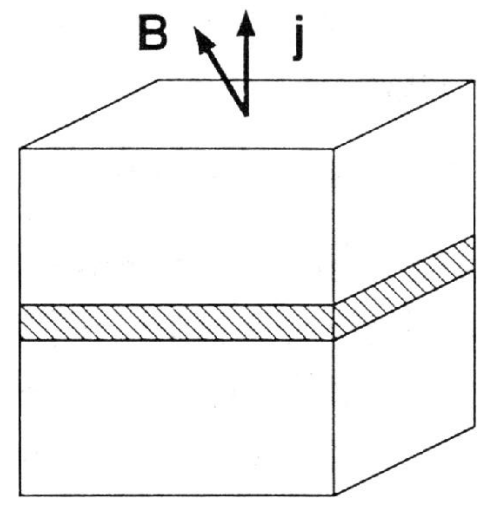

Figure 1: A finite quantum well (shaded region) in a magnetic field $\mathbf{B}$ that is tilted with respect to the vertical direction $\mathbf{x}$. We measure the current across the well as a function of $B$. The undoped well is separated from the doped contacts by undoped spacer layers of the same composition as the contacts.

continuum of propagating states associated with the lowest LL $(n=1)$ but do not mix with this propagating state due to the conservation of the LL index. If we assume that only this lowest LL is populated outside the well then for precisely normal field no resonance occurs, but introducing a small tilt angle with respect to the normal to the interfaces will induce inter-LL scattering at the interfaces. The bound states will then be coupled to the continuum and lead to Fano resonances whose width will increase with the tilt angle $\alpha$.

The experiment must be done at low temperature (so that thermal broadening does not distort the intrinsic lineshape) and in the linear response regime. The latter is possible here because unlike the well-known resonant tunneling devices, this structure contains no large barriers so that one can drive a current without a substantial voltage drop. As has been done in Refs. [2, 3, 4, 5, 6, $7,8,9,10,11,15,16]$, we perform the model calculation at zero temperature. Since the contacts are degenerately doped, we separate them from the well region by undoped spacer layers that can be traversed ballistically. In order to trace out resonance lineshapes, we need a means of varying the energy of the electrons incident on the well, i.e. the Fermi energy $E_{F}$ in the contacts. This can be done [17] by varying the magnitude of the magnetic field, $B$, as shown in Fig. 2. As we increase $B$ with a fixed carrier concentration $\rho$ in the bulk, Landau levels are depopulated due to the growing degeneracy. Once $B$ exceeds some threshhold field $B_{\text {th }}, E_{F}$ will enter the lowest LL. As $B$ is increased further, $E_{F}$ moves from slightly below $E_{2}$ towards the bottom of the lowest LL, $E_{1}$. This causes the initial decrease in $E_{F}$, which is eventually outweighed by the simultaneous increase in $E_{1}$ itself. The results depend somewhat on the degree of spin polarization which is taken into account in Fig. 2. The ratio between spin and LL splitting is measured by the parameter $\eta=\left|g^{*}\right| m^{*} /\left(2 m_{e}\right)$. Here, $g^{*}$ is the electronic $g$-factor, $m_{e}$ and $m^{*}$ denote the bare and effective electron mass, respectively. If $\eta<1$, the Zeeman splitting is smaller than the LL separation so that it is possible to have $E_{F}$ in the lowest LL but with both spin species present. We assume this to be true to simplify what follows (the treatment for $\eta>1$ is similar). The field at which the system is fully spin-polarized is given by

$$
B_{0}^{3}=\frac{\pi \rho^{2}}{4 \eta}\left(\frac{h c}{e}\right)^{3} .
$$

For lower magnetic fields, both spins are present. But we still have $E_{F}<E_{2}$ as long as $B$ is above 


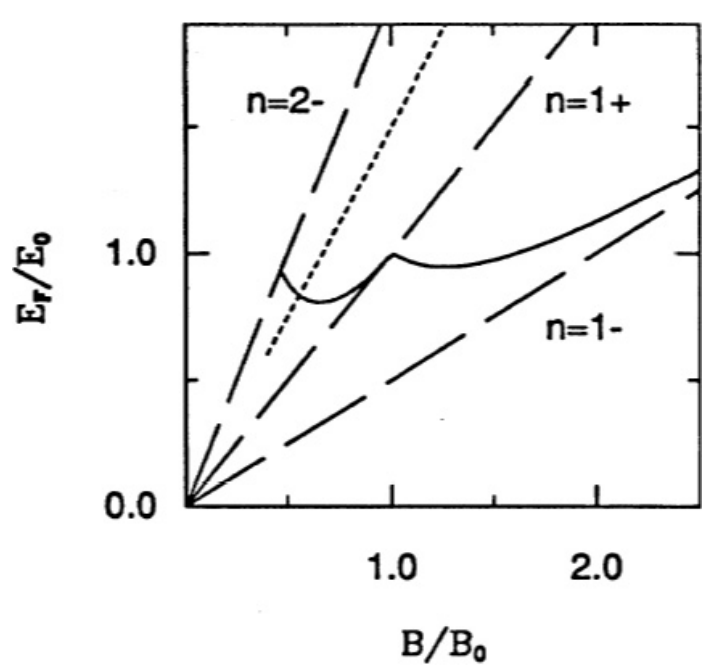

Figure 2: Dependence of the Fermi energy on magnetic field $B$ when $B$ is large enough to bring $E_{F}$ into the lowest LL, i.e. $B>B_{\text {th }}$. The straight dashed lines show the first $(n=1 \pm)$ and second $(n=2-)$ spin-split Landau levels, and the dotted line represents the position of a resonance. The field needed to spin-polarize the system is denoted by $B_{0}$, and the Fermi energy at that magnetic field is $E_{0}$. As shown, $E_{F}$ crosses the resonance as $B$ is varied. We assume $\eta=1 / 3$ in this graph.

the threshold field

$$
B_{t h}^{3}=2 B_{0}^{3} \frac{1}{\eta}\left(1-\frac{1}{2} \eta-\sqrt{1-\eta}\right) .
$$

In units of $B_{0}$, we find that $E_{F}$ as a function of $B$ has the form

$$
E_{F}=\frac{1}{2} \hbar \omega\left\{\begin{array}{lr}
1+\frac{1}{2} \eta\left[\left(\frac{B_{0}}{B}\right)^{3}+\left(\frac{B}{B_{0}}\right)^{3}\right] & \left(B_{t h} \leq B \leq B_{0}\right) \\
1+\eta\left[2\left(\frac{B_{0}}{B}\right)^{3}-1\right] & \left(B \geq B_{0}\right),
\end{array}\right.
$$

with the cyclotron frequency $\omega_{c} \equiv e B / m^{*} c$. The quasibound levels also shift as a function of $B$ but $E_{F}$ will cross all resonances between $E_{2}$ and $E_{1}$ at some $B$. Thus we obtain resonances as a function of magnetic field. The dependence of $E_{F}$ on $B$ becomes more complicated for $B<B_{\mathrm{th}}$, but the resonances occur at these lower fields, too. Since our calculation implies that $\Gamma / \hbar \omega_{c}$ is only a function of the tilt angle $\alpha$, the resonances for a given $\alpha$ will be easiest to resolve if $B$ is large.

To solve the scattering problem for a given $E_{F}$, we first solve the Schrödinger equation of a heterostructure with $x$ as the growth direction and $V(x)$ as the effective potential due to the conduction band modulation. In addition, a tilted magnetic field is applied, $\mathbf{B}=B_{x} \hat{\mathbf{x}}+B_{z} \hat{\mathbf{z}}$. Choosing the gauge $\mathbf{A}=-B_{z} y \hat{\mathbf{x}}+B_{x} y \hat{\mathbf{z}}$, the Hamiltonian is

$$
\begin{aligned}
H= & \frac{1}{2 m^{*}}\left[\left(p_{x}-m^{*} \omega_{z} y\right)^{2}+p_{y}^{2}+\left(p_{z}+m^{*} \omega_{x} y\right)^{2}\right] \\
& +U(x)+\frac{1}{2} g^{*} \mu_{B} B
\end{aligned}
$$

where $\omega_{z} \equiv e B_{z} / m^{*} c$ and $\omega_{x} \equiv e B_{x} / m^{*} c$. In the Zeeman term, we introduced the Bohr magneton, $\mu_{B}=e \hbar / 2 m_{e} c$, which involves the bare electron mass instead of the effective mass. The value of 
$E$ at which to solve the scattering problem is determined by $B$ through Eq. (4), cf. Fig. 2. Since $\left[H, p_{z}\right]=0$, we use the ansatz

$$
\Psi(x, y, z)=\psi(x, y) e^{i k_{z} z}
$$

to obtain a two-dimensional Schrödinger equation for $\psi$ :

$$
\begin{gathered}
{\left[\frac{1}{2 m^{*}}\left\{\left(p_{x}-m^{*} \omega_{z} y\right)^{2}+p_{y}^{2}\right\}\right.} \\
\left.+\frac{1}{2} m^{*} \omega_{x}^{2}\left(y+\frac{\hbar k_{z}}{m^{*} \omega_{x}}\right)^{2}+U(x)\right] \psi=\tilde{E} \psi .
\end{gathered}
$$

Here, $\tilde{E} \equiv E \mp \frac{1}{2} g^{*} \mu_{B} B$. But this is the Schrödinger equation of a parabolic quantum wire, shifted by $\hbar k_{z} /\left(m^{*} \omega_{x}\right)$, with a transverse magnetic field

$$
\mathbf{B}^{\prime}=\nabla \times\left(-B_{z} y \hat{\mathbf{x}}\right)=B_{z} \hat{\mathbf{z}} .
$$

The existence of this mapping to a quantum wire problem went unnoticed in a previous treatment of the quantum well in a tilted magnetic field [19] because a different choice of gauge and coordinate system in Eq. (5) leads to a qualitatively different reduced two-dimensional equation. The current flowing through a $y-z$ plane of the heterostructure is obtained by integrating the $x$ component of the current density over $y$ and $z$. Let the sample dimension along $z$ be $L_{z}$, then

$$
\begin{aligned}
I= & \int d z \int d y\left[\frac { \hbar } { 2 m ^ { * } i } \left(\Psi^{*}(x, y, z) \frac{\partial}{\partial x} \Psi(x, y, z)\right.\right. \\
& \left.-\Psi(x, y, z) \frac{\partial}{\partial x} \Psi^{*}(x, y, z)\right) \\
& \left.-\omega_{z} y|\Psi(x, y, z)|^{2}\right]=L_{z} I^{\prime}
\end{aligned}
$$

where

$$
\begin{aligned}
I^{\prime} \equiv & \int d y\left[\frac { \hbar } { 2 m ^ { * } i } \left(\psi^{*}(x, y) \frac{\partial}{\partial x} \psi(x, y)\right.\right. \\
& \left.\left.-\psi(x, y) \frac{\partial}{\partial x} \psi^{*}(x, y)\right)-\omega_{z} y|\psi(x, y)|^{2}\right]
\end{aligned}
$$

is the current carried by the equivalent quantum wire eigenstate $\psi(x, y)$. We can set $k_{z}=0$ in Eq. (8) Since $I^{\prime}$ is independent of $k_{z}$. This equation has been solved numerically in Refs. [8, 18] for rectangular potentials $U(x)$ (corresponding to a perfectly sharp band profile) and those results need only be reinterpreted in terms of the parameters of our model to yield the results of Fig. 3a. This calculation neglects the band bending at the well interfaces, but we expect this to only affect the absolute position of the resonance and not the lineshape.

The resulting transmission curves as a function of $B$ are shown in Fig. 3 (a) for a particular resonance at various small tilt angles $\alpha$. At an experimental temperature of $30 \mathrm{mK}$, the resolution is limited by $k T \approx 2.6 \mu \mathrm{eV}$. For the narrowest curve in Fig. 3 (a), we find from Eq. (4) an energy difference of $\Delta E_{F} \approx 5.3 \mu \mathrm{eV}$ between valley and peak. The resonances should therefore be experimentally observable even at the small tilt angles we chose here to come close to the ideal lineshape. Across the narrow resonance width we can furthermore approximate Eq. (4) by a linear relation between $B$ and $E_{F}$. If we then rescale the $B$ axis to the variable $\epsilon$ defined below Eq. (1), all resonances should collapse onto a single curve if the Fano asymmetry parameter $q$ is independent of the resonance 

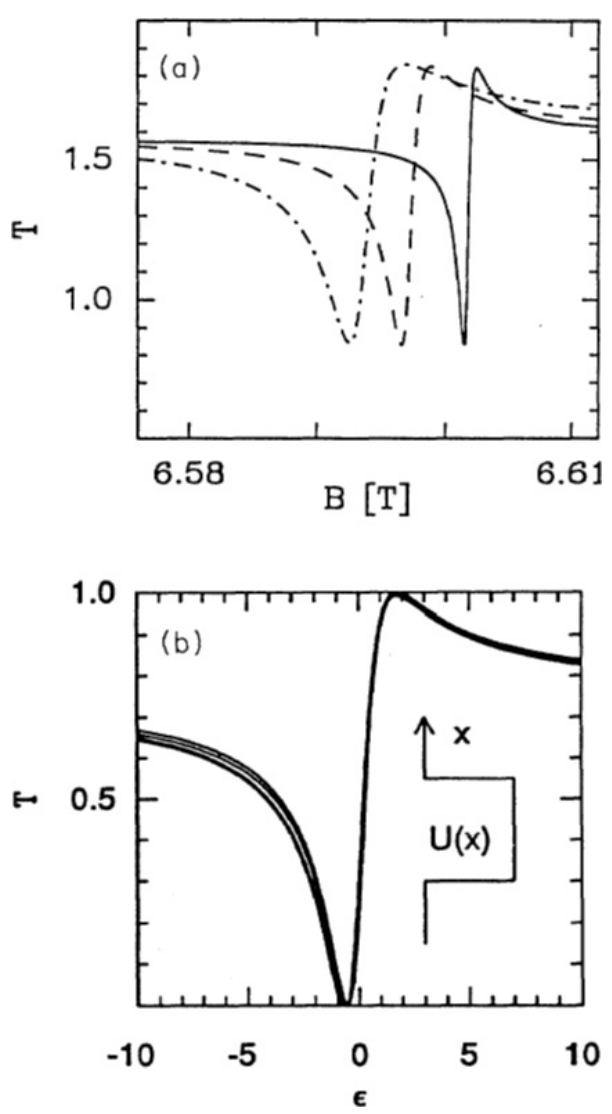

Figure 3: (a) Transmission of a finite well as a function of magnetic field $B$ in units of $B_{0}$. The tilt angles are $\sin \alpha=0.12$ (dashed-dotted), 0.1 (dashed) and 0.08 (solid). We chose a GaInSbInSb-GaInSb structure with well width $L=25.9 \mathrm{~nm}$ and depth $V=168 \mathrm{meV}$. The large electronic $g$-factor and small effective mass of this compound combine to give $\eta \approx 1 / 3$, cf. [20]. (b) The same resonances plotted in reduced units, $\epsilon$ being defined as below Eq. ( 1); the Fano asymmetry parameter is $q=0.577$. Over the width of these narrow resonances, $E_{F}$ is proportional to $B$ while $\left|t^{b}\right|^{2}$ and $\Gamma$ are independent of $B$. Inset: effective potential in the growth direction.

lifetime. As shown in Fig. 3 (b), this is indeed the case. We also subtracted out a constant baseline arising from the fact that both spin channels contribute to the transmission but one of them is not on resonance (and thus simply adds a nonresonant background contribution). Note that the resonant variation of the transmission is exactly unity as predicted in Ref. [17].

Thus the experiment, if successful, would not only observe the Fano lineshape in electronic transport for the first time, but could also provide a novel test of basic predictions of scattering theory for isolated resonances.

We acknowledge $\mathrm{R}$. Wheeler for the important suggestion of tuning through resonance with a magnetic field. We also thank M. Büttiker and M. Reed for helpful discussions. This work was supported by ARO grant no. DAAH04-93-G0009. 


\section{References}

[1] C. Weisbuch and B. Vinter, Quantum Semiconductor Structures (Academic Press, San Diego, 1991)

[2] M. Büttiker, Phys. Rev. A 30, 1982 (1984)

[3] R. L. Schult, H. D. Wyld and D. G. Ravenhall, Phys. Rev. B 41, 12760 (1990)

[4] P. F. Bagwell, Phys. Rev. B 41, 10354 (1990)

[5] H. U. Baranger, Phys. Rev. B 42, 11479 (1990)

[6] W. Porod, Zhi-an Shao and C. S. Lent, Appl. Phys. Lett. 61, 1350 (1992)

[7] P. F. Bagwell and R. K. Lake, Phys. Rev. B 46, 15329 (1992)

[8] J. U. Nöckel, Phys. Rev. B 46, 15348 (1992)

[9] E. Tekman and P. Bagwell, Phys. Rev. B 48, 2553 (1993)

[10] Z. Shao, W. Porod, C. S. Lent, Phys. Rev. B 49, 7453 (1994)

[11] S. A. Gurvitz and Y. B. Levinson, Phys. Rev. B 47, 10578 (1993)

[12] P. L. McEuen, B. W. Alphenaar, R. G. Wheeler and R. N. Sacks, Surf. Sci. 229, 312 (1990).

[13] G. Timp, in: Physics of Nanostructures, J. H. Davies and A. R. Long (eds.), SUSSP proceedings, Institute of Physics Publishing, Bristol and Philadelphia (1992)

[14] J. A. Simpson and U. Fano, Phys. Rev. Lett. 11, 158 (1963)

[15] D. Y. K. Ko and J. C. Inkson, Semicond. Sci. Technol. 3, 791 (1988)

[16] D. Z. Y. Ting and T. C. McGill, Phys. Rev. B 47, 7281 (1993)

[17] J. U. Nöckel and A. D. Stone, unpublished

[18] J. U. Nöckel, Phys. Rev. B 45, 14225 (1992)

[19] G. Marx and R. Kümmel, J. Phys. Cond. Matter 3, 8237 (1991)

[20] K. H. Hellwege (ed.), Landolt-Börnstein. Numerical Data and Functional Relationships in Science and Technology, Vol. 17a (Springer Verlag, Berlin, 1982) 